Aus dem Institut für gerichtliche und soziale Medizin der Universität Kiel (Direktor: Prof. Dr. W. Hallermann)

\title{
EIN BEITRAG ZUR FORENSISCHEN VERWERTBARKEIT DER RH-UNTERGRUPPEN
}

\author{
von \\ W. Hallermann und V. Nagel
}

Nachdem wir (1) unter Hinweis auf eigene Familienuntersuchungen und auf die beobachtete Verteilung der Rhesus-Faktoren ihre theoretische Bedeutung für forensische Vaterschaftsverfahren, insbesondere bezüglich der Ausschlusschancen, erörtert haben, ist es nunmehr möglich, an Hand eines eigenen, inzwischen beträchtlich angewachsenen forensischen Untersuchungsmaterials die erwarteten den beobachteten Werten gegenüberzustellen, diese Ergebnisse statistisch auszuwerten, dadurch die Bedeutung der Rh-Untergruppenbestimmung in praxi aufzuzeigen und die Frage der Zuverlässigkeit der angewandten Untersuchungsmethode kritisch zu prüfen. Wir folgen damit dem Beispiel von Läuppi (2), der - allerdings nach einer anderen Methode - bereits im vorigen Jahr auf Grund eigener Untersuchungen die forensische Verwertbarkeit der Rhesus-Faktoren überzeugend darstellte.

Eine Uebersicht über das eigene Material und über die zahlenmässige Verteilung der Vaterschaftsausschlüsse auf die einzelnen Blutgruppensysteme bzw.- eigenschaften gibt Tabelle 1.

Bei insgesamt 859 Parteien (Spalte a) mit 1251 beteiligten Männern (Spalte b) wurden neben den $\mathrm{ABO}$, und MN-Eigenschaften die Rhesus-Faktoren $\mathrm{Rh}^{\prime}, \mathrm{Rh}^{\circ}, \mathrm{Rh}^{\prime \prime}$ und $\mathrm{hr}^{\prime}$ unter Verwendung eigener und amerikanischer Testseren nach der bereits in der früheren Mitteilung ausführlich beschriebenen Methode bestimmt. Für spätere Vergleiche war es notwendig, die Mindestzahl der zu Unrecht beschuldigten Männer festzustellen (Spalte c). Zu ihnen zählen wir auch der Einfachheit halber die am Prozess als Mehrverkehrszeugen Beteiligten. Bei den Mehrmannsachen ${ }^{2}$ ergibt sich diese Zahl aus der jeweiligen Anzahl der beteiligten Männer, bei den Einmannsachen ${ }^{1}$ wurde sie von der Zahl der ausgeschlossenen Männer abgeleitet, d. h., die Ausschlusszahl wurde verdoppelt, da nach der hiesigen Merkmalsverteilung insgesamt 49,8\% der zu Unrecht beschuldigten Männer ausgeschlossen werden können. Unter den 537 in den Einmannsachen in Anspruch genommenen Männern finden sich danach also 31,3\% "Nichtväter». Dieser

\footnotetext{
1 Einmannsachen: Ein Mann (Beklagter) am Prozess beteiligt.

2 Mehrmannsachen: Mehrere Männer (ein Beklagter und ein bzw. mehrere Zeugen) am Prozess beteiligt.
} 
Acta Geneticae Medicae et Gemellologiae

Tab. $x$ - Uebersicht über das gesamte Untersuchungsmaterial

\begin{tabular}{|c|c|c|c|c|c|c|c|c|c|c|c|c|}
\hline & \multirow{2}{*}{ 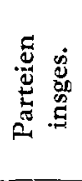 } & \multirow{2}{*}{ 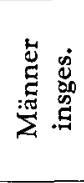 } & \multirow{2}{*}{ 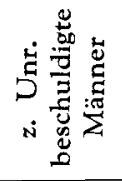 } & \multirow{2}{*}{ 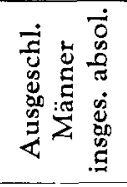 } & \multirow{2}{*}{ 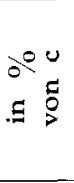 } & \multicolumn{7}{|c|}{$\begin{array}{l}\text { Verteilung der Ausshlüsse auf die } \\
\text { Blutgruppeneigenschaften }\end{array}$} \\
\hline & & & & & & $\mathrm{ABO}$ & $M N$ & $\mathbf{R h}$ & $\mathrm{Rh}^{\prime}$ & $\begin{array}{c}\mathrm{Rh}^{\prime} \mathrm{u} \\
\mathrm{hr}^{\prime}\end{array}$ & $\mathrm{Rh}^{\circ}$ & $\mathrm{Rh}^{\prime \prime}$ \\
\hline & a & b & c & d & e & f & $\mathrm{g}$ & h & i & $\mathbf{j}$ & k & 1 \\
\hline Einmannsachen & 537 & 537 & $\begin{array}{c}(168) \\
(31,3 \%)\end{array}$ & 84 & 50,6 & 23 & 35 & 38 & 7 & 28 & 1 & 10 \\
\hline Zweimannsachen & 267 & 534 & 267 & 135 & 50,6 & 50 & 53 & 60 & 10 & 40 & 4 & 23 \\
\hline Dreimannsachen & 44 & 132 & 88 & 41 & 46,6 & 13 & 11 & 26 & 8 & 19 & 3 & 7 \\
\hline Viermannsachen & 7 & 28 & 21 & 12 & 57,1 & 5 & 5 & 4 & 0 & 2 & 0 & 2 \\
\hline Fünfmannsachen & 4 & 20 & 16 & 8 & 50,0 & 0 & 4 & 5 & 0 & 5 & 0 & 0 \\
\hline $\begin{array}{l}\text { Mehrmannsachen } \\
\text { insges. }\end{array}$ & 322 & 714 & 392 & 196 & 50,0 & 68 & 73 & 95 & 18 & 66 & 7 & 32 \\
\hline $\begin{array}{l}\text { Summe } \\
\text { Gesamtmaterial }\end{array}$ & 859 & 1251 & 560 & $\begin{array}{c}280 \\
(22,4 \%)\end{array}$ & & 91 & 108 & 133 & 25 & 94 & 8 & 42 \\
\hline
\end{tabular}

Prozentsatz ersche int im Vergleich zu anderen Angaben verhältnismässig niedrig. Läuppi berichtet, dass etwa $43 \%$ der Frauen den falschen Mann als Vater bezeichnen. Nach Wiethold (3) soll sich in etwa nur 50\% der wirkliche Vater unter den Verdächtigten befinden. Die Frage, ob vielleicht durch eine zu geringe Auschlusshäufigkeit im RhSystem unser niedriger Prozentsatz bedingt ist, muss aber beim Vergleich mit der die anderen Systeme betreffenden Ausschlusshäufigkeit verneint werden. Dabei dürfen wir die einfach und sicher zu bestimmenden klassischen Blutgruppen und die MN-Faktoren als gültigen Masstab nehmen. Es zeigt sich dann nach entsprechender Berechnung, dass die beobachtete Ausschlusshäufigkeit (in \% von den zu Unrecht beschuldigten Männern) von der erwarteten Ausschlusschance in den 3 Systemen in etwa gleich geringem Masse abweicht.

\begin{tabular}{lccc}
\multicolumn{2}{c}{ beobachtet } & erwartet & Differenz \\
ABO : & $13,7 \%$ & $16,6 \%$ & $-2,9 \%$ \\
MN: & $20,8 \%$ & $18,8 \%$ & $+2,2 \%$ \\
Rh: & $22,6 \%$ & $26,1 \%$ & $-3,5 \%$
\end{tabular}

Ausserdem geht aus den Angaben der erwähnten Autoren auch nicht eindeutig hervor, ob es sich dort nur um Einmannsachen handelt, oder ob in ihrem Material auch Mehrmannsachen enthalten sind. In letzterem Falle, der der wahrscheinlichste ist, wäre die Differenz zwischen ihren und unseren Ergebnissen daraus zu erklären. 
Wie aus Spalte d) der Tabelle 1 zu ersehen ist, wurden insgesamt etwa 280 Männer ( $=22,4 \%$ von der Gesamtzahl der beteiligten Männer) ausgeschlossen. Diese Zahl entspricht nicht der Summe aller aus den einzelnen Systemen ausgeschlossenen Männer (Spalten $\mathrm{f}, \mathrm{g}, \mathrm{h}$ ), weil in der Gesamt-Ausschlusszahl die Ausschlusskombinationen also gleichzeitig aus 2 oder 3 Systemen erfolgte Ausschlüsse - nur einfach, in den Spalten $f$, $g$ und $h$ aber unter jedem System getrennt aufgeführt sind.

Ferner wurden die jeweiligen Ausschlusszahlen ins prozentuale Verhältnis zu den in Spalte $c$ eingezeichneten Mindestzahlen der zu Unrecht beschuldigten Männer gesetzt (Spalte e). Wie bereits erwähnt, beträgt die für unser Material gültige Gesamtausschlusschance bei Bestimmung der klassischen Blutgruppen, der MN. Faktoren und der RhesusFaktoren $\mathrm{Rh}^{\prime}, \mathrm{Rh}^{\circ}, \mathrm{Rh}^{\prime \prime}$ und $\mathrm{hr}^{\prime}$ 49,8\%. Diese Zahl ergibt sich bei Anwendung der von Geppert und Koller (4) bzw. Hirszfeld (5) angegebenen Methoden aus den ausgezählten Häufigkeiten. Diese betragen für die hiesige Bevölkerung nach Angaben von Schöner (6)

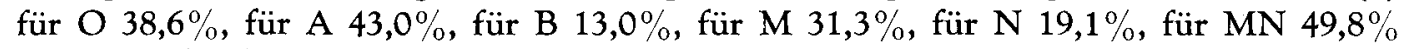
und nach den bereits mitgeteilten eigenen Untersuchungen von insgesamt 1200 Personen für $\mathrm{Rh}^{\prime} 70,5 \%$, für $\mathrm{Rh}^{\circ} 84,0 \%$ und für $\mathrm{Rh}^{\prime \prime} 72,0 \%$.

Es fällt auf, dass die für die summierten Mehrmannsachen errechnete Ausschlusshäufigkeit von $50 \%$ fast genau der theoretischen Ausschlusschance von $49,8 \%$ der zu Unrecht beschuldigten Männer entspricht. Unter der Voraussetzung, dass unsere Bestimmungen zuverlässig sind, kann man daraus schliessen, dass sich in der Regel unter den am Prozess beteiligten Männern auch der leibliche Kindesvater befindet. Anderenfalls müsste die wirkliche Anzahl der zu Unrecht beschuldigten Männer die feststehende und für unsere Berechnungen eingesetzte Mindestzahl deutlich übersteigen, und infolgedessen würden die beobachteten Vaterschaftsausschlüsse weit mehr als 50\% dieses Mindestwertes betragen. Die hier an der Summe der Mehrmannsachen getroffene Feststellung gilt, wie aus Spalte e zu ersehen ist, in gleicher Weise auch für die Zweimannsachen. Die Zahlen der Drei- bis Fünfmannsachen sind wegen des zu geringen Umfanges des jeweiligen Materials für sich alleine in diesem Sinne nicht zu verwerten. Immerhin sind aber auch hier die Abweichungen auffallend gering bzw. minimal.

Die absolute Verteilung der Vaterschaftsausschlüsse auf die einzelnen Systeme bzw. auf die bestimmten $4 \mathrm{Rh}$-Faktoren ist aus den Spalten $\mathrm{f}$ bis 1 der Tabelle $1 \mathrm{zu}$ ersehen. Die grosse Bedeutung des Rh-Systems für die Ausschlussfrequenz tritt auch an diesem grossen Material wieder eindrucksvoll in Erscheinung: Während aus dem klassischen Blutgruppensystem 91 und aus dem MN-System 108 Männer ausgeschlossen wurden, betrug die Ausschlusszahl bei alleiniger Anwendung der Rh-Untergruppen-Bestimmung 133 Männer. Dabei steht der Wert der Diagnose von $\mathrm{Rh}^{\prime}$ und $\mathrm{hr}^{\prime}$ an hervorragender Stelle, wie der Vergleich der Spalten i bis 1 zeigt. Denn alleine bei Verwendung von Anti-Rh' und Anti-hr' könnten 94 der insgesamt 133 aus dem Rh-System ausgeschlossenen Männer erfasst werden. Auch hier sind übrigens die kombinierten Rh-Ausschlüsse unter den einzelnen Merkmalen getrennt aufgeführt, sodass die Summen der Spalten i bis 1, die in Spalte $\mathrm{h}$ eingezeichneten Werte übersteigen.

Um neben diesen mehr orientierenden Vergleichen nunmehr die eigentliche und wesentlichste Fragestellung unseres Themas, nämlich das Verhältnis zwischen der beob- 
Acta Geneticae Medicae et Gemellologiae

Tab. 2 - Veteilung der Vaterschaftsausschlüsse auf die Blutgruppeneigenschaften

\begin{tabular}{|c|c|c|c|c|c|c|c|}
\hline \multicolumn{8}{|c|}{ A. Gesamtmaterial } \\
\hline & \multicolumn{3}{|c|}{ absolut } & \multicolumn{4}{|c|}{ in $\%$ von den gesamten Ausschlüissen (280) } \\
\hline & $\begin{array}{c}\text { beob. } \\
\text { a }\end{array}$ & $\begin{array}{c}\text { erw. } \\
\mathbf{b}\end{array}$ & $\begin{array}{c}\text { Diff. } \\
\mathfrak{c}\end{array}$ & $\begin{array}{c}\text { beob. } \\
\text { d }\end{array}$ & $\begin{array}{c}\text { erw. } \\
\mathrm{e}\end{array}$ & $\begin{array}{l}\text { Diff. } \\
\quad f\end{array}$ & $\begin{array}{c}3 \text { facher mittl. } \\
\text { Fehler }\end{array}$ \\
\hline $\mathrm{ABO}$ & 91 & 93,2 & $-2,2$ & 32,5 & 33,3 & $-0,8 \%$ & $\pm 8,40 \%$ \\
\hline MN & 108 & 104,4 & $+3,6$ & 38,6 & 37,3 & $+1,3 \%$ & $\pm 8,73 \%$ \\
\hline $\mathrm{Rh}$ & 133 & 147,3 & $-14,3$ & 47,5 & 52,6 & $-5,1 \%$ & $\pm 8,94 \%$ \\
\hline $\mathrm{Rh}^{\prime}$ & 25 & 23 & $+2,0$ & 8,9 & 8,2 & $+0,7 \%$ & $\pm 5,1 \%$ \\
\hline $\mathrm{Rh}^{\prime}$ u. $\mathbf{h r}^{\prime}$ & 94 & 104,7 & $-10,7$ & 33,6 & 37,4 & $-3,8 \%$ & $\pm 8,46 \%$ \\
\hline $\mathrm{Rh}^{\mathrm{o}}$ & 8 & 8,4 & $-0,4$ & 2,9 & 3,0 & $-0,1 \%$ & $\pm 3,03 \%$ \\
\hline $\mathrm{Rh}^{\prime \prime}$ & 42 & 44,5 & $-2,5$ & 15,0 & 15,9 & $-0,9 \%$ & $\pm 6,39 \%$ \\
\hline
\end{tabular}

B. Mehrmannsachen

\begin{tabular}{l|c|c|c|c|c|c|c}
\hline $\mathrm{ABO}$ & 68 & 65,1 & $\% 2,9$ & 17,3 & 16,6 & $+0,7 \%$ & $\pm 4,00 \%$ \\
$\mathrm{MN}$ & 73 & 73 & 0 & 18,6 & 18,6 & 0 & - \\
$\mathrm{Rh}$ & 95 & 102,3 & $-7,3$ & 24,2 & 26,1 & $-2,1 \%$ & $\pm 6,48 \%$ \\
\hline $\mathrm{Rh}^{\prime}$ & 18 & 16,1 & $+1,9$ & 4,6 & 4,1 & $+0,5 \%$ & $\pm 3,18 \%$ \\
$\mathrm{Rh}^{\prime}$ u. $\mathbf{h r}^{\prime}$ & 66 & 72,9 & $-6,9$ & 16,8 & 18,6 & $-1,8 \%$ & $\pm 6,00 \%$ \\
$\mathrm{Rh}^{\circ}$ & 7 & 5,9 & $+1,1$ & 1,9 & 1,5 & $+0,4 \%$ & $\pm 2,05 \%$ \\
$\mathbf{R h}^{\prime \prime}$ & 32 & 31,0 & $+1,0$ & 8,2 & 7,9 & $+0,3 \%$ & $\pm 4,17 \%$ \\
\hline insgesamt & 196 & 195,2 & 0,8 & 50,0 & 49,8 & $+0,2 \%$ & $\pm 7,59 \%$
\end{tabular}

achteten und der erwarteten Verwertbarkeit darzustellen, war es notwendig, zunächst die erwarteten Ausschlusszahlen für die einzelnen Systeme und vor allem für die Rh-Untergruppen zu berechnen, und zwar den jeweiligen erwarteten absoluten und prozentualen Anteil der Systeme bzw. der Merkmale an der Gesamtzahl der Ausschlüsse von 280 Männern (cfr. Tab. 2).

Als Grundlage für diese Berechnungen dienten neben der bereits erwähnten, für unsere Bevölkerung zutreffenden theoretischen Gesamt-Ausschlusschance von 49,8\% die spezifizierten Ausschlusserwartungen. Sie ergeben sich aus der oben angegebenen Merkmalsverteilung und betragen bei alleiniger Bestimmung der jeweiligen Merkmale für $\mathrm{ABO} 16,6 \%$, für $\mathrm{MN} 18,6 \%$, für $\mathrm{Rh}\left(\mathrm{Rh}^{\prime}, \mathrm{hr}^{\prime}, \mathrm{Rh}^{\circ}\right.$ und $\left.\mathrm{Rh}^{\prime \prime}\right) 26,1 \%$, für $\mathrm{Rh} \mathbf{h}^{\prime} 4,1 \%$, für $\mathrm{Rh}^{\prime}$ und $\mathrm{hr}^{\prime} 18,6 \%$, für $\mathrm{Rh}^{\circ} 1,5 \%$ und für $\mathrm{Rh}^{\prime \prime} 7,9 \%$ der zu Unrecht beschuldigten Männer. Da nach unseren Berechnungen aber insgesamt 49,8\% aller zu Unrecht beschul- 
digten Männer ausgeschlossen werden können, mussten diese Prozentzahlen zu 49,8 ins Verhältnis gebracht und zusätzlich die erhaltenen prozentualen Werte (Spalte e) in absoluten Zahlen ausgedrückt werden (Spalte b). Es war jetzt nur noch zu prüfen, ob die festgestellten Differenzen zwischen den beobachteten und erwarteten Zahlen bzw. Prozentsätzen (Spalten d bis f) echte, d. h. statistisch gesicherte Werte sind, oder ob sie noch in den Bereich des mittleren Fehlers und damit in die zufällige Streuungsbreite fallen.

Während die so errechneten Differenzen für das klassische und das MN-System sehr klein sind und $-2,2(-0,8 \%)$ bzw. $+3,6(+1,3 \%)$ betragen, weicht die beobachtete Ausschlusszahl im Rh-System von der erwarteten etwas stärker ab. Die absolute Differenz beträgt hier nämlich - 14,3. Allerdings liegt auch diese Abweichung von $-5,1 \%$ noch im Bereiche der Fehlerbreite. Erst wenn sie den 3 fachen mittleren Fehler von $\pm 8,94 \%$ überschreiten würde, wäre sie significant und liesse auf eine mangelnde Zuverlässigkeit der $\mathrm{Rh}$-Untergruppenbestimmung schliessen. Andererseits ist es aber nicht ausgeschlossen, dass diese Abweichung der beobachteten von der erwarteten Ausschlusshäufigkeit im Rh-System durch vereinzelte Fehlbestimmungen entstanden ist, denn in dem ausgewerteten Material sind auch unsere ersten Rh-Untergruppenbestimmungen einbegriffen. Bekanntlich erfordert aber eine fehlerlose Anwendung dieser diffizilen Untersuchungsmethode grosse Erfahrung und vor allem eine genaue Kenntnis der angewandten Testseren. Es sei in diesem Zusammenhange nur auf die von Läuppi zitierte Auffassung des amerikanischen Committees on Medicolegal Problems bezüglich der Zuverlässigkeit von Rhesus-Bestimmungen hingewiesen: "In the opinion of this committee the Rh-Hr tests constitue a reliable addition to the other blood tests in cases of disputed parentage, provided, of course, that the person who carries out the tests is an expert who has had extensive experience with the performance of such tests ". Möglicherweise hat sich in der ersten Zeit auch bei uns die allgemein bekannte Tatsache der schwierigeren serologischen Fassbarkeit von hr' auf die Ausschlusshäufigkeit ausgewirkt, denn, wie die differenzierte Verteilung der Vaterschaftsausschlüsse auf die einzelnen Rh-Gene eindeutig zeigt, sind bei der isolierten Bestimmung von $\mathrm{Rh}^{\prime}, \mathrm{Rh}^{\circ}$ und $\mathrm{Rh}^{\prime \prime}$ die Abweichungen minimal, während die Differenz zwischen beobachteter und erwarteter Ausschlusshäufigkeit bei kombinierter Bestimmung von $\mathrm{hr}^{\prime}$ und $\mathrm{Rh}^{\prime}$ deutlich grösser ist (S. Tabelle $2 \mathrm{~A}$ ). Jedoch liegt auch diese Abweichung von -3,8\% noch eindeutig im Bereiche des 3 fachen mittleren Fehlers von $8,46 \%$, sodass von einer mangelhaften Verwertbarkeit auch hier nicht gesprochen werden kann.

Um das Bild zu vervollständigen, wurden schliesslich noch die Mehrmannsachen gesondert ausgewertet (s. Tabelle $2 \mathrm{~B}$ ), wobei wir als für die Relationen massgeblichen Wert die Mindestzahl der zu Unrecht beschuldigten Männer gewählt haben. Als erwartete Grössen wurden hier die prozentualen und daraus berechneten absoluten Werte eingesetzt, wie sie sich aus der Merkmalsverteilung in Bezug auf die Gesamtzahl der zu Unrecht beschuldigten Männer ergeben (s. o.). Entsprechend den oben, an Hand der Gesamtübersicht (Tabelle 1) getroffenen Feststellungen ist auch hier in der differenzierten Auswertung ein erwartungsgerechtes Verhältnis der Systeme und Merkmale untereinander und eine weitgehende Uebereinstimmung zwischen den beobachteten und erwarteten Zahlen zu finden. Das bedeutet, dass auch im Hinblick auf den Anteil der 
einzelnen Systeme und Merkmale die sicher zu bestimmende Mindestzahl der zu Unrecht beschuldigten Männer im wesentlichen ihre tatsächliche Anzhal darstellt. In Uebereinstimmung mit den Ergebnissen der Tabelle $2 \mathrm{~A}$ sind auch hier die Differenzen minimal bzw. unbedeutend. Sie liegen sämtlich im Bereiche des 3 fachen mittleren Fehlers. Die Abweichungen im $\mathrm{Rh}$-System und vor allem bei alleiniger Diagnose von $\mathrm{Rh}^{\prime}$ und $\mathrm{hr}^{\prime}$ sind im Vergleich zu den am Gesamtmaterial erhobenen Differenzen weitaus geringer. Die isolierte Bestimmung der $\mathrm{Rh}$-Faktoren $\mathrm{Rh}^{\prime}, \mathrm{Rh}^{0}$ und $\mathrm{Rh}^{\prime \prime}$ hat hier sogar im Hinblick auf die positiven Abweichungen einen geringen Ueberschuss an Vaterschaftsausschlüssen ergeben.

Insgesamt hat sich also auch auf Grund unseres eigenen Materials die Rh-Untergruppenbestimmung als zuverlässige und den Erwartungen entsprechende Vaterschaftsausschlussmethode erwiesen. Dabei ist u. E. der Diagnose des Rh-Merkmals hr' bzw. der Herstellung der entsprechenden Anti-Seren ganz besondere Sorgfalt zu widmen. Es wäre begrüssenswert, wenn grundsätzlich jede Bestimmung der Rhesus-Faktoren von 2 unabhängig voneinander arbeitenden Untersuchern vorgenommen würde. Damit stimmen wir der Ansicht Läuppi's in vollem Umfange zu. U. E. dürfte die Verwirklichung dieser begründeten Forderung nicht an der Kostenfrage scheitern. Dies gilt insbesondere für die Vaterschaftsausschlüsse und in hervorragendem Masse für die $\mathrm{Rh}$ Untergruppenbestimmungen als Beweismittel im Strafverfahren (Meineid oder falsche uneidliche Aussage). Es wäre zu erwägen, ob in diesen letztgenannten Fällen nicht jeder Gutachter von sich aus das Gericht auf die Notwendigkeit eines Zweitgutachtens hinweisen sollte, wie es ja auch bei den MN-Ausschlüssen geschieht. Hier einen weitgehend übereinstimmenden Standpunkt einzunehmen, sollte eine vornehmliche Aufgabe aller serologisch tätigen Gutachter sein. Dabei ist zu bedenken, dass das Ergebnis der Blutgruppenbestimmung in Straf- und Zivilprozessen entscheidend für die Urteilsfindung des Richters sein dürfte. Der Richter muss aber die Ueberzeugung haben, dass die Resultate richtig sind. Die mit Recht engen Voraussetzungen (z. B. im Zivilrecht, der Begriff des "offenbar unmöglich») verlangen zwingend die höchstmögliche Sicherheit. Ein auf einer falschen Blutgruppenbestimmung fundiertes Urteil lässt sich nicht leicht revidieren und pflegt dann eine Erschütterung des Beweiswertes des ganzen Blutgruppenverfahrens mit sich zu bringen. Diese Gefahr lässt sich durch eine zwingende Vorschrift eines $\mathrm{Z}_{\text {weit- }}$ gutachtens sehr erheblich verringern, sie muss deshalb trotz der dadurch entstehenden Mehrkosten verlangt werden.

\section{Zusammenfassung}

An Hand eines sehr umfangreichen eigenen forensischen blutgruppenserologischen Materials (859 Parteien mit 1251 beteiligten Männern) wird die Frage der Verwertbarkeit bzw. der Zuverlässigkeit der Rh-Untergruppenbestimmung kritisch erörtert. Die beobachteten Ausschlusshäufigkeiten entsprechen weitgehend den erwarteten Werten. Die festgestellten Abweichungen sind entweder minimal oder liegen zumindest noch eindeutig im Bereiche der zufälligen Streuungen. Auf die Notwendigkeit der WiederholungsUntersuchungen - insbesondere bei Vaterschaftsausschlüssen und im Rahmen des Strafverfahrens - wird hingewiesen. 


\section{Literatur}

1. Nagel, V., Hoffmann, M., Prokop, O.: Act. gen. med. et gemellol. II, 2, 126-38, 1953

2. LÄUpPI, E.: Vortrag, Tagung d. Dtsch. Ges. für gerichtl. und soziale Med. 1952.

3. Wiethold, F.: zit. nach Schöner.

4. Geppert, Koller.: Erbmathematik, Quelle und Meyer-Verlag Leipzig, 1938.

5. Hirszfeld, L.: Schweiz. Ztschr. f. allgem. Path. u. Bakteriol. XV, 1952, 3, 257-280.

6. Schöner, M.: Dissertation, Kiel, 1950.

\section{RIASSUNTO}

Sulla scorta d'un ricchissimo materiale relativo a ricerche forensi gruppo-specifiche (859 comparse in giudizio con I25 I partecipanti) viene posta criticamente la questione sull'utilizzazione $e$, in particolare, sull'attendibilità della determinazione del sottogruppo $\mathrm{Rh}$.

La frequenza nell'esclusione, che si è potuta osservare, è, grosso modo, corrispondente ai valori che si attendevano. Le variazioni riscontrate o sono minime, o rientrano almeno nell'ambito dell'incidenza casuale.

Gli AA. rilevano che si pone in tutti i casi la necessità di ripetere gli esami, specialmente in quei casi in cui si tratti di decidere della paternità o di procedere penalmente.

\section{RÉSUMÉ}

Prenant pour base le matériel médico-légal très nombreux de nos séroréactions (859 partis avec I251 hommes intéressés), nous discutons sur l'applicabilité et sur la certitude des sous-groupes du facteur Rhésus. Les exclusions observées correspondent à peu près aux résultats attendus. Quant aux différences constatées, elles sont ou insignifiantes, ou, du moins, elles restent dans la sphère des variations du hasard. Nous renvoyons à la nécessité des examens répétées, surtout au cours du procès criminel et de l'exclusion de la paternité.
SUMMARY

The suitability and reliability of the Rh-Antigens is being discussed in details in the light of a great number of forensic serologic tests carried out in our Institute in cases of discussed parentage (859 contracting parties, I25I males involved).

The frequency of paternity exclusions observed does greatly conform with the frequency expected. Differences noted are either very small or are in the range of standard deviation.

Authors emphasize that it is necessary to repeat the test especially if a criminal case is concerned or if an accused father can be excluded. 